\title{
Proceso de negocios de la cadena de suministro en las empresas de fluidos de perforación
}

\author{
Supply chain business process in drilling fluids companies
}

Processo de negócios da cadeia de suprimentos em empresas de fluidos de perfuração

Recibido: octubre 2018

Arbitrado: noviembre 2018

Publicado: enero 2019
《I Margareth Schmid

schmid_522@hotmail.com

ORCID: 0000-0002-0607-8947

Universidad del Zulia, Núcleo Costa Oriental del lago, Venezuela

\section{RESUMEN}

El objetivo de la investigación, es describir el proceso de negocios de la cadena de suministro utilizado en las empresas petroleras de fluidos de perforación. La investigación es descriptiva, con diseño no experimental, transeccional y de campo. Se empleó un censo poblacional, para un total de 25 sujetos informantes, pertenecientes a las cinco empresas de fluidos de perforación de pozos petroleros con taladros activos en la Costa Oriental del Lago. Para recolectar la información, se aplicó un cuestionario conformado por 20 ítems, validado por expertos y con un 0,96 de confiabilidad según el coeficiente Alfa Crombach. El análisis de los datos se realizó mediante la media aritmética. Se concluye alta utilización de la administración de las relaciones con el cliente, administración del servicio al cliente, gestión de la demanda, cumplimiento de los pedidos, gestión del flujo de fabricación, desarrollo y comercialización del producto, devoluciones como procesos de negocios.

Palabras clave: Administración del cliente; administración del servicio; cadena de suministro; cumplimiento de pedidos; desarrollo y comercialización; devoluciones; gestión de demanda; gestión del flujo de fabricación, proceso de negocios

ABSTRACT

RESUMO

The objective of the research is to describe the supply chain business process used in drilling fluid oil companies. The research is descriptive, with a nonexperimental, transectional and field design. A population census was used, for a total of 25 informant subjects, belonging to the five oil well drilling fluid companies with active drills on the Eastern Coast of the Lake. To collect the information, a questionnaire consisting of 20 items was applied, validated by experts and with a reliability of 0.96 according to the Alpha Crombach coefficient. The data analysis was performed using the arithmetic mean. High utilization of customer relationship management, customer service management, demand management, order fulfillment, manufacturing flow management, product development and marketing, returns as business processes is concluded.

Key words: Client administration; service administration; supply chain, order fulfillment; development and commercialization; returns; demand management; manufacturing flow management, business process
O objetivo da pesquisa é descrever o processo de negócios da cadeia de suprimentos utilizado em empresas de óleo de fluido de perfuração. A pesquisa é descritiva, com desenho não experimental, transversal e de campo. Foi utilizado um censo populacional, para um total de 25 sujeitos informantes, pertencentes às cinco empresas de fluidos de perfuração de poços de petróleo com perfurações ativas na Costa Leste do Lago. Para a coleta das informações, foi aplicado um questionário composto por 20 itens, validado por especialistas e com confiabilidade de 0,96 de acordo com o coeficiente Alpha Crombach. A análise dos dados foi realizada pela média aritmética. Alta utilização de gerenciamento de relacionamento com o cliente, gerenciamento de serviço ao cliente, gerenciamento de demanda, atendimento de pedidos, gerenciamento de fluxo de fabricação, desenvolvimento de produto e marketing, devoluções conforme os processos de negócios são concluídos.

Palavras-chave: Administração de clientes; administração de serviços; cadeia de suprimentos, atendimento de pedidos; desenvolvimento e comercialização; retorna; gestão da demanda; gerenciamento de fluxo de manufatura, processos de negócios 


\section{INTRODUCCIÓN}

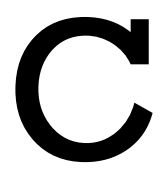

on la finalidad de generar una estrategia de cooperación en entornos turbulentos, las empresas requieren continuamente incrementar su nivel de repuesta ante los cambios que presenta la demanda en el nuevo contexto de la economía global. De manera que, es importante que éstas conozcan cuáles son las tendencias y prioridades sobre las que tendrán que trabajar para lograr su permanencia en el medio donde se desenvuelven; no obstante, el adecuado desempeño de una organización y de su sistema logístico, ya no depende de sí misma, sino también de sus proveedores, distribuidores, clientes y todos aquellos que forman parte de su cadena de suministro.

Al respecto, para Chase y otros (2005), la cadena de suministro, conocida en inglés como "Supply Chain", es una cadena de proveedores, fábricas, almacenes, centros de distribución y detallistas a través de los cuales se adquieren las materias primas, se transforman y se envían al cliente, en ocasiones se gestiona proceso devolutivo, dependiendo el sector y el producto.

En este sentido se puede inferir, que la cadena de suministros, agrupa todos los procesos, que inician a partir de la procura y adquisición de la materia prima, continúan con la elaboración del producto, hasta la entrega de los productos terminados en el lugar adecuado, en el tiempo indicado, y en la cantidad deseada. Involucra procesos de intercambio de información, materiales, servicios, productos semiterminados, productos terminados, operaciones de post acabado, de posventa y de logística inversa, todo ello, para lograr una ventaja competitiva a la vez de que consiguen optimizar los recursos económicos y operativos de la empresa.

En el contexto venezolano, la cadena de suministro es relativamente nueva, muchas empresas no trabajan con alianzas que permitan establecer un vínculo de sociedad operacional entre proveedor, comprador y cliente; en muchos casos el departamento de compras cumple una función más transaccional que estratégica y actúa aislado del área logística.

Ahora bien, en el estado Zulia, específicamente en la Costa Oriental del Lago, Venezuela, existen empresas del ramo petrolero trabajando con gestión de cadena de suministro como una nueva manera de ver las cosas, que ha hecho evidente, el enorme potencial que representa un sistema bien diseñado para avanzar en el cumplimiento de las metas estratégicas de la empresa.

Así, la aplicación de la cadena de suministro a obteniendo resultados satisfactorios, pues hay una visión más clara de lo necesario, cómo y cuándo, con una participación activa dentro de los procesos de todos los elementos integrantes de la cadena; sin embargo, un diseño incorrecto en su cadena de suministro, las transacciones de información, materiales y recursos, resultarían complicadas, atrasando las operaciones, y generando elevados costos operativos, originando grandes pérdidas para la organización, desde pérdida monetarias, hasta la credibilidad de sus clientes estratégicos.

Bajo esta óptica, y dado que la integración de los procesos en una cadena de suministro mejora su nivel de competitividad, generando productos en la cantidad, condiciones y momento 
requerido, a un bajo costo, se desarrolla el presente artículo, cuyo objetivo se centró en describir el proceso de negocios de la cadena de suministro utilizado en las empresas petroleras de fluidos de perforación en la Costa Oriental del Lago.

\section{Proceso de negocios de la cadena de suministro}

Según el Council Logistic Management (2007), para el éxito de la cadena de suministro se requiere cambiar las actividades funcionales por actividades integrales relacionadas con los procesos determinantes de la cadena. Tradicionalmente, los proveedores y clientes en una operación de suministro actúan de manera recíproca como entidades desconectadas que reciben flujos de información de manera esporádica.

En la gestión de la cadena de suministro se requiere que la información fluya continuamente para producir el flujo más adecuado sobre los bienes. Es importante recordar esto debido a que el enfoque en la gestión de la cadena de suministro tiene como base el cliente, se requiere de información precisa y oportuna de los procesos para obtener de los sistemas una respuesta rápida respondan a los frecuentes cambios y fluctuaciones en la demanda. Una vez controlada la incertidumbre de la demanda del cliente, los procesos industriales y la actuación del proveedor, son básicos en la eficacia de la cadena de suministro.

Para Markovic y Pereira (2007) el proceso de negocio en la cadena de suministro, se entiende como la aplicación de técnicas para modelar, gestionar y optimizar los procesos de negocio de la organización. Partiendo de que el proceso es la forma natural de organización, el modelado de los procesos permite establecer un flujo de trabajo entre funciones, para tratar de conseguir que con el trabajo mancomunado y funcional se capturen los requerimientos del negocio para obtener un mejor entendimiento, esto facilita la comunicación así como identificar las mejoras en los procesos con el objetivo de conseguir los objetivos de la organización, las expectativas y requerimientos de los clientes, de una forma eficaz y eficiente.

Sobre los postulados descritos, se considera que la cadena de suministro y los procesos de negocio, enmarcan componentes que deben recibir atención, estos abarcan una amplia gama desde estratégico hasta operacional, flujo físico hasta flujo de información, estructuras tangibles hasta las estructuras organizacionales y culturales. Componentes similares se encuentran en la literatura de reingeniería de procesos, estos forman parte de un compendio estratégico operacional que van de la mano del trabajo realizado por el recurso humano así como también de la planificación exhaustiva de cada una de las actividades diseñadas e implementadas.

Visto así, en este punto se considera la administración de las relaciones con el cliente, administración del servicio al cliente, gestión de la demanda, cumplimiento de los pedidos, gestión del flujo de fabricación, desarrollo y comercialización del producto, así como las devoluciones como aspectos inherentes al proceso de negocios de la cadena de suministros. 


\section{Administración de las relaciones con el cliente}

De acuerdo con Lambert (2008) el primer paso en la integración de la administración de la cadena de suministro es identificar los clientes o grupo de clientes que pueden ser considerados como críticos o importantes en la misión comercial de la compañía. Se establece los acuerdos específicos del servicio con este grupo y al mismo tiempo, se trabaja con los clientes más alejados en la cadena, identificando y eliminando fuentes de variabilidad de la demanda.

Para Heizer y Render (2004) a los clientes no les importa cómo se administra o utiliza su información en las organizaciones, lo que les importa es obtener un servicio excelente e inmediato, por esto surge la administración de la relación con los clientes (CRM). CRM es una combinación de proceso de negocios y tecnología que pretende comprender a los clientes de una compañía desde una visión multifacética.

La única manera en que una organización pueda tener éxito es enfocándose hábilmente en las necesidades del cliente. Para conservar a los mejores clientes, la gerencia debe concentrar sus energías en crear rápida y eficientemente nuevos canales de entrega, capturar cantidades masivas de datos sobre los clientes y relacionar todo para crear una experiencia única.

Según Greenberg (2008) es una estrategia de marketing destinada a construir proactivamente una preferencia en los consumidores por una determinada organización, lo cual suele resultar en unos mayores índices de retención de esos consumidores y en un rendimiento económico mayor, el CRM es una estrategia orientada al largo plazo, que requiere inversiones tecnológicas y estratégicas que dan fruto cuando el cliente acaba dándose cuenta de que realmente la compañía lo comprende y le satisface mejor que la competencia. En cierto sentido, se trata de una redefinición de la compañía desde el punto de vista del cliente.

De acuerdo con los planteamientos descritos, la administración de las relaciones con el cliente es un proceso que comprende llevar a cabo evaluaciones de desempeño, los cuales permiten analizar el nivel de servicio proporcionado a los clientes, la satisfacción de estos, entre otros disimiles factores que sirven de mucha ayuda al momento de evaluar al consumidor, su comportamiento y hábitos de consumo, que son de utilidad para crear nuevos productos o mejorar los ya existentes.

\section{Administración del servicio al cliente}

De esta manera, Lambert (2008) el servicio al cliente proporciona una fuente de información muy importante y es una actividad relevante que permite administrar los acuerdos sobre las características y especificaciones de los productos o servicio comprometidos.

A partir de una mayor interrelación con el área de producción y los sistemas de distribución de la organización, el departamento de servicio al cliente permite proporcionar información en tiempo real sobre los compromisos de sus envíos, fechas y disponibilidad del producto. En un sistema de cadena de suministro, las funciones de este departamento incluyen una orientación al cliente sobre el uso de los productos que comercializan. 
Aunado a ello, para Cox (2012) clientes satisfechos es el resultado deseado de toda estrategia de administración de cadenas de suministro, es por ello que el proveedor de productos o servicios debe conocer y entender quién es el cliente intermedio y quién es el cliente final y cuales son necesidades.

Para Greenberg (2008) en la industria de los operadores logísticos es común encontrar una visión muy limitada respecto del concepto de cliente y más limitada aún respecto de los costos y beneficios directos e indirectos de una buena atención a los clientes. Para muchos especialistas captar un cliente cuesta cinco a seis veces más que mantenerlo. Mientras que conseguir un cliente nuevo requiere de acciones comerciales que deben madurar; la mantención de clientes es básica, simple y muy económica.

\section{Gestión de la demanda}

Según Vollmann y otros (2005) los sistemas de gestión de las demandas suelen recurrir a los puntos de venta y bases de datos de los clientes, esto permite una mejor eficiencia del flujo físico de mercancías a lo largo de la cadena de suministro, en cuanto a las necesidades de comercialización y programas de producción, se deben coordinar sobre la base de una empresa extendida, es decir, considerando las necesidades de los clientes y los proveedores. En otras palabras, la demanda del cliente y la capacidad de producción, se sincroniza para manejar inventarios integralmente.

De acuerdo con Ballou (2004) la gestión de la demanda se encarga de predecir y regular los ciclos de consumo, adaptando la producción a los picos de mayor exigencia para asegurar que el servicio se sigue prestando de acuerdo a los tiempos y niveles de calidad acordados con el cliente, comúnmente cuando mejor es el producto o el servicio mayor demanda genera.

Así las cosas, la gestión de la demanda se encarga de predecir y regular los ciclos de consumo, adaptando la producción a los picos de mayor exigencia para asegurar que el servicio se sigue prestando de acuerdo a los tiempos y niveles de calidad acordados con el cliente. Por lo general, cuanto mejor funciona un servicio, mayor demanda genera. Ésta, a su vez, provoca exigencias de capacidad que los responsables compensan, como es natural, incrementando los activos del servicio. Se genera así un ciclo de consumo-producción en el que el consumo es un estímulo positivo para la producción y viceversa.

Desde la siguiente perspectiva se considera la gestión de la demanda como un elemento clave en la eficaz administración de la cadena de suministro, y durante el proceso de gestión de la demanda se deben equilibrar los requisitos del cliente con la capacidad de suministro de la empresa, intentando determinar qué y cuándo comprarán, es decir pronosticando lo que sucederá a pesar de los patrones irregulares que se pueden topar en un ámbito de mercado. 


\section{Cumplimiento de los pedidos}

En consonancia con Lambert (2008) la clave de una eficiente cadena de suministro está en el cumplimiento de los requerimientos del cliente. Bajo esta óptica, el logro de una alta proporción de cumplimiento de los pedidos se vuelve importante. Por esta razón, para el cumplimiento de los pedidos se requiere de un proceso eficiente de integración de los planes de fabricación, distribución y transporte.

Para lograr lo anterior, se debe desarrollar pactos con los miembros clave de la cadena de suministro y en especial con los transportistas para cumplir con los requisitos del cliente y así reducir el costo total de distribución. Como objetivo de la investigación, se propone desarrollar un proceso de gestión desde el proveedor hasta varios segmentos de clientes.

En este orden de ideas, Coyle y otros (2013) consideran que el cumplimiento de pedidos consiste en las actividades involucradas en el despacho y embarque de los pedidos del cliente. Es importante porque tiene un impacto directo sobre el tiempo transcurrido, entre el momento en que el cliente coloca un pedido hasta que lo recibe. A esto también se le llama tiempo de entrega del pedido. Los cuatro procesos o actividades básicas para el cumplimiento o tiempo de entrega son transmisión, procesamiento, preparación y envío.

De acuerdo con Ballou (2004) el cumplimiento de pedidos viene dado por el esfuerzo mancomunado que realizan los departamentos de una organización con el fin de satisfacer a tiempo las demandas y los requerimientos de la cartera de clientes, para ello se debe vigilar además de verificar celosamente todo el proceso de la cadena de suministro hasta llegar el producto al consumidor, así conocer si se está cumpliendo con el tiempo estimado y si se abastece de manera acorde la demanda existente, para ello los autores recomiendan el uso de la tecnología para canalizar los pedidos así como las entregas de manera más oportuna.

Por lo tanto, se considera que una organización podría considerar la adición de tecnología para el procesamiento y la transmisión de los pedidos a fin de reducir ambos procesos a un menor tiempo, esto le permitiría usar un modo de transportación de bajo costo para cumplir con el compromiso prontamente y ofrecer tiempos de entrega más bajos para obtener con ello una ventaja competitiva.

\section{Gestión del flujo de fabricación}

De acuerdo con Lambert (2008) en las empresas tradicionales la gestión de los flujos de fabricación sigue un proceso común: producir, almacenar y entregar los productos terminados al sistema de distribución de acuerdo con las previsiones históricas. Este esquema de fabricación, los productos son elaborados bajo un estricto programa de producción, sin embargo, una característica común de este tipo de sistemas, es que se presenten inventarios innecesarios y excesivos, los cuales generalmente causan altos costos. 
En la operación y administración de la cadena de suministro, el producto se elabora con base en las necesidades del cliente, los procesos de fabricación se flexibilizan para responder a cambios en la comercialización, mediante la instalación de sistemas dinámicos que puedan adaptarse a la consolidación de los diferentes productos (customization).

Es importante señalar que en la operación de la cadena de suministro, los pedidos se procesan con sistemas "justo a tiempo" en cantidades mínimas, con prioridades definidas por la fecha de entrega y de acuerdo a los requerimientos. La gestión del flujo de fabricación en este nuevo ambiente ha traído consigo cambios en el proceso de fabricación de tiempos de ciclo más cortos, mejoras en el servicio al cliente.

En este orden de ideas, Coyle, y otros (2013) afirman que el alma de cualquier sistema de producción es el proceso de manufactura, un proceso de flujo con dos componentes importantes: materiales e información. El flujo físico de los materiales se puede ver, pero el flujo de información es intangible y más difícil de rastrear. Ambos tipos siempre han estado presentes pero en el pasado, se daba poca importancia al flujo de información. En la actualidad la nueva tecnología de la información ha dado otra forma a los sistemas de producción, de tal manera que el flujo de información es sumamente necesario.

Según Miranda, (2008) el flujo de fabricación contempla el transcurrir del material por todo el proceso productivo y de fabricación, el material fluye desde el proveedor al sistema de producción para convertirse en inventario de materia prima, después se mueve a la planta donde tiene lugar la conversión del material, este se mueve a través de diferentes procesos de transformación en las estaciones de trabajo hasta alcanzar el producto finalizado.

Así que, para esta investigación la necesidad del consumidor, se refleja en lo que se produce, en tal sentido se considera que la operatividad de la cadena de suministro y los productos deben realizarse según lo exigido por el consumidor, a su vez debe ser entregado de manera oportuna; así mismo, la gestión tecnológica ha permitido reducir los tiempos en el flujo de fabricación permitiendo de esta manera tener a disponibilidad el producto terminado en un menor tiempo.

\section{Desarrollo y comercialización del producto}

Lambert (2008) hace énfasis que en la gestión de la cadena de suministro, los clientes y proveedores se integran para desarrollar nuevos productos, con el propósito de reducir los tiempos de comercialización. Cuando el ciclo de vida de los bienes se acorta, éstos se lanzan al mercado en períodos más cortos para mantenerse competitivos.

Para Kotler y Armtrong (2012), es imprescindible el hecho de que una empresa sin importar su tamaño logre sus objetivos, de contemplar la estrategia global, desarrollando la misma en pequeñas acciones que actúen tanto, en su mercado externo (entorno), como en el interno, es por ello donde para innovar en la creación de nuevos productos o la mejora de los existentes se debe tomar muy en cuenta las necesidades y deseos latentes y estudiar el mercado al cual se desea 
llegar, en torno a la cadena de suministro debe poseerse estrategias que permitan cumplir con la demanda del mercado y usar los mecanismos de distribución más idóneos, así estos no encarezcan los costos y permitan la inmediatez a las manos del cliente o consumidor.

En este orden de ideas, Stanton y otros (2004) indican que el desarrollo y la comercialización del producto implica una serie de factores de índole tanto interno como externos, dónde deben considerarse tendencias del mercado, oferta/demanda, cantidad de competidores, ventajas competitivas entre otros, este pues es un proceso que requiere muchísima coordinación, planificación, seguimiento y control por parte de la persona responsable del producto e interrelación con diversos departamentos internos así como también proveedores externos a la empresa.

La investigación considera que el desarrollo y comercialización de un producto debe coordinarse con el área de atención al cliente para identificar la articulación con estos, se deben seleccionar materiales y proveedores para el suministro así como, desarrollar tecnología para facilitar la fabricación e integración de los flujos en la cadena de suministro.

\section{Devoluciones}

De acuerdo con Alet (2007) la administración del canal de devoluciones como proceso de negocios, ofrece la misma oportunidad para lograr una ventaja competitiva sustentable en la cadena de suministro desde una perspectiva de ventas, a su vez, para Lambert (2008) el tiempo de ciclo requerido para volver activar el bien a un estado útil es una medida de velocidad que denomina "devolución disponible". Esta medida es particularmente importante para aquellos productos dónde se presentan clientes que exigen el reemplazo inmediato en caso que el producto falle.

En este sentido, el estudio determina que la administración eficaz del procesamiento de las devoluciones posibilita la identificación de oportunidades para mejorar la productividad y el descubrimiento de nuevos proyectos, tal vez sea así para algunos casos, no obstante, la logística de las devoluciones es una solución parcial el cual tiene como último fin la eliminación de ineficiencias y controversias innecesarias que surgen durante las actividades de la cadena de suministro.

Por otro lado, Castellanos (2015) considera que una gestión eficiente de las devoluciones puede representar, además de una situación crítica entre el fabricante y el distribuidor o el cliente, una pérdida importante en el volumen de ventas, un elemento clave podría estar en la acción rápida por parte del distribuidor para retornar dichas devoluciones y no permitir acumulaciones de lotes que generen mayor perdidas, también se puede practicar la opción de "no devolución" el cual es muy usada el caso de la industria alimenticia donde se pacta de forma contractual un porcentaje de los productos averiados, así se reducen costos de transporte en devoluciones y otras modificaciones o cargas por concepto logístico y administrativo.

De esta forma, este estudio consideró importante manejar eficientemente la gestión de devoluciones, si bien es cierto, se debe manejar controles de calidad que permita no incurrir en 
devoluciones, a pesar de que existan factores que no puedan manejarse, es por ello que cuando ocurran las devoluciones debe manejarse un proceso que permita no acumular productos ociosos ni se incurran en gastos adicionales que disminuyan la rentabilidad de la empresa.

\section{MÉTODO}

L a investigación es de carácter descriptiva, con diseño no experimental, transeccional y de campo. Se empleó un censo poblacional, conformado por 25 sujetos informantes, pertenecientes a las cinco empresas de fluidos de perforación de pozos petroleros con taladros activos en la Costa Oriental del Lago: Halliburton, Esvenca, Lovenca, Inpark Drilling y Saman Servicios Petroleros.

Para recolectar la información, se aplicó un cuestionario conformado por 20 ítems, con escala de frecuencia. La validez se realizó a través del juicio de cinco expertos, y para calcular su confiabilidad se empleó el método del Coeficiente Alfa de Cronbach, cuyo resultado fue de 0,96, ubicándose en una categoría de muy alta confiabilidad según el baremo establecido.

El análisis de los datos se realizó mediante la estadística descriptiva, a través del estudio de la media aritmética, utilizando el baremo establecido para la interpretación del estadístico, cuyos valores se refleja en la tabla 1.

Tabla 1. Categoría de análisis para la interpretación del promedio

\begin{tabular}{ccc}
\hline DIMENSIÓN & RANGO & NIVEL DE RESPUESTA \\
\hline & $4.21-5.00$ & Muy alta utilización \\
Proceso de negocios & $3.41-4.20$ & Alta utilización \\
& $2.61-3.40$ & Moderada utilización \\
& $1.81-2.60$ & Baja utilización \\
& $1.00-1.80$ & No hay utilización \\
\hline
\end{tabular}

\section{RESULTADOS Y DISCUSIÓN}

$\mathrm{E}$ n lo referente, al indicador administración de las relaciones con el cliente, sus resultados se muestran en la tabla 1, cuya media de 4,00 e indica alta utilización. Este nivel del indicador es explicado por los resultados obtenidos en los ítems utilizados y permite inferir que las empresas bajo estudio analizan proactivamente las preferencias de los consumidores. Sin embargo, por su nivel de alta y no así de muy alta utilización, representa una oportunidad de mejora para quienes gestionan la cadena de suministro. 
Tabla 2. Administración de las relaciones con el cliente

\begin{tabular}{llcl}
\hline Ítems & $\overline{\boldsymbol{X}}$ & \multicolumn{1}{c}{ Categoría } \\
\hline $\begin{array}{l}\text { 1. Se cumple con las exigencias del cliente intermediario } \\
\text { 2. Se cumple con las exigencias del consumidor final }\end{array}$ & 3,96 & Alta utilización \\
$\begin{array}{l}\text { 3. Se evalúan factores de comportamiento de consumo para } \\
\text { introducción de nuevos productos. }\end{array}$ & 3,12 & Alta utilización \\
\hline Total & $\mathbf{4 , 0 0}$ & Alta utilización \\
\hline
\end{tabular}

De esta manera se tiene que cumplen de forma alta las exigencias del cliente intermediario $(3,96)$, con las exigencias del consumidor final $(4,12)$, así como, de evaluar los factores de comportamiento de consumo para introducción de nuevos productos $(3,92)$.

Estos resultados confirman, en alto grado, lo expuesto por Lambert (2008), el cual establece como primer paso para la integración de la administración de la cadena de suministro la identificación de los clientes o grupo de clientes que pueden ser considerados como críticos o importantes en la misión comercial de la compañía. También se alcanza alta coincidencia con Heizer y Render (2004), quienes la visualizan como una combinación de proceso de negocios y tecnología que pretende comprender a los clientes de una compañía desde una visión multifacética.

Por lo tanto, con esta investigación se consideró la administración de las relaciones con el cliente un proceso que comprende llevar a cabo evaluaciones de desempeño, los cuales permiten analizar el nivel de servicio proporcionado a los clientes, la satisfacción de estos, entre otros disimiles factores que sirven de mucha ayuda al momento de evaluar al consumidor, su comportamiento y hábitos de consumo, que son de utilidad para crear nuevos productos o mejorar los existentes.

Seguidamente, los datos en la tabla 2, corresponden al indicador administración del servicio al cliente, en ésta se apreció una media de 4,03 implicando alta utilización de este indicador como proceso de negocios de cadena de suministro que se analiza. Permitiendo inferir que en las empresas analizadas, los clientes satisfechos es el resultado de las estrategias de administración de cadenas de suministro, y que deben ser objetos de análisis al no alcanzar el nivel de muy alta utilización.

Tabla 3. Administración del servicio al cliente

\begin{tabular}{|c|c|c|}
\hline Ítems & $\overline{\bar{X}}$ & Categoría \\
\hline $\begin{array}{l}\text { 4. Existe un feedback entre el cliente y la empresa que permite } \\
\text { conocer la satisfacción }\end{array}$ & 4,28 & Muy alta utilización \\
\hline 5. Se atiende todo el mercado & 3,60 & Alta utilización \\
\hline $\begin{array}{l}\text { 6. Se crean estrategias para satisfacer las necesidades de los } \\
\text { consumidores }\end{array}$ & 4,20 & Alta utilización \\
\hline Total & 4,03 & Alta utilización \\
\hline
\end{tabular}


Especialmente se nota que las empresas dan muy alta utilización a la existencia de un feedback entre el cliente y la empresa que permite conocer la satisfacción $(4,28)$, y con alta utilización se atiende todo el mercado $(3,60)$ y se crean estrategias para satisfacer las necesidades de los consumidores $(4,03)$.

Este indicador cumple la teoría de Lambert (2008), donde el servicio al cliente proporciona una fuente de información importante y es una actividad relevante que permite administrar los acuerdos sobre las características y especificaciones de los productos o servicio comprometidos.

Adicional a esto, se confirma en la investigación que la administración del servicio con el cliente el departamento encargado permite proporcionar información en tiempo real sobre los compromisos de sus envíos, fechas y disponibilidad del producto, de esta manera se pueden cumplir con las necesidades o deseos mediante una acorde cadena de suministro que permita disponer de la mercancía en el tiempo justo y solicitado por el cliente.

La tabla 3, contiene los datos referidos al indicador gestión de la demanda. En ellos se puede apreciar como la media se ubicó en 3,79 indicando alta utilización. De esta manera, se infiere que en las empresas bajo estudio, se predicen y regulan los ciclos de consumo, adaptando la producción para asegurar que el servicio se preste de acuerdo a los tiempos y niveles de calidad acordados con el cliente, pero al no ubicarse en el nivel de muy alta utilización, representa una oportunidad de mejora para quienes gestionan la cadena de suministro.

Tabla 4. Gestión de la demanda

\begin{tabular}{llcc}
\hline Ítems & $\overline{\boldsymbol{X}}$ & Categoría \\
\hline $\begin{array}{l}\text { 7. La empresa mantiene un correcto equilibrio entre oferta y } \\
\text { demanda. }\end{array}$ & 3,68 & Alta utilización \\
8. Se sincroniza el manejo de los inventarios de manera correcta & 4,08 & Alta utilización \\
9. Se cumple a cabalidad con la demanda existente en el mercado & 3,60 & Alta utilización \\
\hline Total & $\mathbf{3 , 7 9}$ & Alta utilización \\
\hline
\end{tabular}

Ello se debe fundamentalmente a que con alta utilización la empresa mantiene un correcto equilibrio entre oferta y demanda $(3,68)$, se sincroniza el manejo de los inventarios de manera correcta $(4,08)$ y se cumple a cabalidad con la demanda existente en el mercado $(3,60)$.

Los resultados se ajustan, a la opinión de Ballou (2004), para quien la gestión de la demanda se encarga de predecir y regular los ciclos de consumo, adaptando la producción a los picos de mayor exigencia para asegurar que el servicio se sigue prestando de acuerdo a los tiempos y niveles de calidad acordados con el cliente, comúnmente cuando mejor es el producto o el servicio mayor demanda genera.

En este sentido, el proceso de gestión de la demanda debe equilibrar los requisitos del cliente con la capacidad de suministro de la empresa, intentando determinar qué y cuándo comprarán, es 
decir, pronosticando lo que sucederá a pesar de los patrones irregulares que se pueden topar en un ámbito de mercado.

Con relación al indicador cumplimiento de los pedidos, en la tabla 5 muestra la información recabada, observándose un valor para la media de 3,80 indicando alta utilización, por lo que a criterio de esta investigación estos resultados representan una oportunidad de mejora al no posicionarse en muy alta utilización, en cuanto alcanzar que el objetivo sea desarrollar un proceso de gestión desde el proveedor hasta varios segmentos de clientes.

Tabla 5. Cumplimiento de los pedidos

\begin{tabular}{lccc}
\hline Ítems & $\overline{\boldsymbol{X}}$ & Categoría \\
\hline $\begin{array}{l}\text { 10. Es eficiente el proceso de integración de los planes de perforación } \\
\text { 11. Se cumple a cabalidad con los pedidos realizados por los miembros de la } \\
\text { cadena }\end{array}$ & 3,96 & Alta utilización. \\
$\begin{array}{l}\text { 12. Se maneja el proceso de entrega justo a tiempo para satisfacer al } \\
\text { consumidor. }\end{array}$ & 3,84 & Alta utilización \\
\hline \multicolumn{1}{c}{ Total } & $\mathbf{3 , 8 0}$ & Alta utilización \\
\hline
\end{tabular}

El valor obtenido en esta media, es producto de la alta utilización dado a todos los reactivos medidos; así se cumple de manera alta con que: es eficiente el proceso de integración de los planes de perforación $(3,96)$, se cumple a cabalidad con los pedidos realizados por los miembros de la cadena $(3,60)$, se maneja el proceso de entrega justo a tiempo para satisfacer al consumidor $(3,84)$.

Corresponde este resultado, de forma alta, a lo que describe Lambert (2008) cuando afirma que la clave de una eficiente cadena de suministro está en el cumplimiento de los requerimientos del cliente. Bajo esta óptica, el logro de una alta proporción de cumplimiento de los pedidos se vuelve importante. Por esta razón, para el cumplimiento de los pedidos se requiere de un proceso eficiente de integración de los planes de fabricación, distribución y transporte.

De igual manera se validó, que toda la organización podría considerar la adición de tecnología para el procesamiento y la transmisión de los pedidos a fin de reducir ambos procesos a un menor tiempo, esto permitirá usar un modo de transportación de bajo costo para cumplir con el compromiso prontamente y ofrecer tiempos de entrega más bajos para obtener con ello una ventaja competitiva.

Los valores presentados en la tabla 6 corresponden al indicador gestión del flujo de fabricación. En los mismos se apreció como la media se ubicó en 4,20 indicando alta utilización. Tal resultado obedece a que se tiene alta presencia de todas las actividades pertinentes al proceso productivo y de fabricación, representando una oportunidad de mejora al no ubicarse en muy alta utilización. 
Tabla 6. Gestión del flujo de fabricación

\begin{tabular}{lccc}
\hline Ítems & $\overline{\boldsymbol{X}}$ & Categoría \\
\hline $\begin{array}{l}\text { 13. Se elabora un programa para la cadena de suministro } \\
\begin{array}{l}\text { 14. Se realiza una red acorde en el tránsito de los materiales por } \\
\text { todo el proceso de perforación }\end{array}\end{array}$ 4,36 & $\begin{array}{c}\text { Muy alta utilización } \\
\text { Muy alta utilización }\end{array}$ \\
$\begin{array}{l}\text { 15. La empresa utiliza un sistema automatizado en flujo de } \\
\text { perforación }\end{array}$ & 3,92 & Alta utilización \\
\hline Total & $\mathbf{4 , 2 0}$ & Alta utilización
\end{tabular}

De tal forma, se da muy alta utilización al que se elaboró un programa para la cadena de suministro $(4,36)$, y se realiza una red acorde en el tránsito de los materiales por todo el proceso de perforación $(4,32)$, no obstante pero no menos importante con alta utilización la empresa utiliza un sistema automatizado en flujo de perforación $(3,92)$.

Este resultado, coincide con lo que expresa Lambert (2008) para quien en las empresas tradicionales la gestión de los flujos de fabricación sigue un proceso común: producir, almacenar y entregar los productos terminados al sistema de distribución de acuerdo con las previsiones históricas. Este esquema de fabricación, los productos son elaborados bajo un estricto programa de producción, sin embargo, una característica común de este tipo de sistemas, es que se presenten inventarios innecesarios y excesivos, los cuales generalmente causan altos costos.

Asimismo, para la investigación, se consideró que la operatividad de la cadena de suministro y los productos deben realizarse según lo exigido por el consumidor a su vez ser entregado de manera oportuna, la gestión tecnológica ha permitido reducir los tiempos en el flujo de fabricación permitiendo de esta manera tener a disponibilidad el producto terminado en un menor tiempo.

Seguidamente, se presenta la tabla 7, la cual muestra la información correspondiente al indicador desarrollo y comercialización del producto; según estos datos, se aprecia para el indicador una media de 3,85 indicando alta utilización del indicador. En este sentido, se basa en los resultados obtenidos, que en la gestión de la cadena de suministro, los clientes y proveedores se integran para desarrollar nuevos productos, con el propósito de reducir los tiempos de comercialización.

Tabla 7. Desarrollo y comercialización del producto

\begin{tabular}{lcc}
\hline Ítems & $\overline{\boldsymbol{X}}$ & \multicolumn{1}{c}{ Categoría } \\
\hline $\begin{array}{l}\text { 16. La empresa realiza continuas inversiones en creación de nuevos } \\
\text { procesos de perforación }\end{array}$ & 3,80 & Alta utilización \\
$\begin{array}{l}\text { 17. La empresa cumple con los tiempos de comercialización de } \\
\text { manera efectiva }\end{array}$ & 3,52 & Alta utilización \\
$\begin{array}{l}\text { 18. La empresa toma en cuentas las sugerencias del cliente para la } \\
\text { mejora continua del proceso de perforación }\end{array}$ & 4,24 & Muy alta utilización \\
\hline Total & $\mathbf{3 , 8 5}$ & Alta utilización \\
\hline
\end{tabular}


Sin embargo, por su carácter de alta utilización y no de muy alta utilización, representa una oportunidad de mejora para quienes gestionan la cadena de suministro, sustentado en el nivel de alta utilización al referir los encuestados que la empresa realiza continuas inversiones en creación de nuevos procesos de perforación $(3,80)$, y cumplen con los tiempos de comercialización de manera efectiva $(3,52)$, así mismo con muy alta utilización toma en cuentas las sugerencias del cliente para la mejora continua del proceso de perforación $(4,24)$.

De acuerdo a estos resultados, se logró validar, lo planteado por Stanton y otros (2004) quienes indican que el desarrollo y la comercialización del producto implica una serie de factores de índole tanto interno como externos, dónde deben considerarse tendencias del mercado, oferta/demanda, cantidad de competidores, ventajas competitivas entre otros, este pues es un proceso que requiere coordinación, planificación, seguimiento y control por parte de la persona responsable del producto e interrelación con diversos departamentos internos así como también proveedores externos a la empresa.

De manera se debe seleccionar materiales y proveedores para el suministro así como también desarrollar tecnología para facilitar la fabricación e integración de los flujos en la cadena de suministro.

La tabla 8, presenta los resultados para el indicador devoluciones, en esta se aprecia un valor para la media de 4,10 indicando alta utilización, de esta forma, la administración eficaz del procesamiento de las devoluciones posibilita la identificación de oportunidades para mejorar la productividad y el descubrimiento de nuevos proyectos. Ahora bien su alta utilización y no muy alta utilización representa una oportunidad de mejora para quienes gestionan la cadena de suministro en las empresas bajo estudio.

Tabla 8. Devoluciones

\begin{tabular}{lcc}
\hline Ítems & $\overline{\boldsymbol{X}}$ & Categoría \\
\hline $\begin{array}{l}\text { 19. La tasa de devoluciones por incumplimiento del proceso en el } \\
\text { tiempo establecido es baja }\end{array}$ & 4,00 & Alta utilización \\
\begin{tabular}{l} 
20. Las pérdidas por devoluciones la asumen la empresa \\
\multicolumn{2}{c}{ Total }
\end{tabular} & 4,20 & Alta utilización \\
\hline
\end{tabular}

Observándose, en tal sentido, que se otorga alta utilización a que la tasa de devoluciones por incumplimiento del proceso en el tiempo establecido es baja $(4,00)$, y las pérdidas por devoluciones la asume la empresa $(4,20)$.

Estos resultados concuerdan, con lo expresado por Alet (2007), el cual, considera la administración del canal de devoluciones como proceso de negocios, que ofrece la misma oportunidad para lograr una ventaja competitiva sustentable en la cadena de suministro desde una perspectiva de ventas, a su vez coincide con lo expuesto por Lambert (2008) quien expone que el 
tiempo de ciclo requerido para volver activar el bien a un estado útil es una medida de velocidad que denomina "Devolución disponible". Esta medida es particularmente importante para aquellos productos dónde se presentan clientes que exigen el reemplazo inmediato en caso que el producto falle.

Por lo tanto, se debe manejar un proceso que permita no acumular productos ociosos ni se incurran en gastos adicionales que disminuyan la rentabilidad de la empresa.

Se puede apreciar en la tabla 9 el resumen para la dimensión proceso de negocios, la cual muestra un valor para la media de 3,97 indicando alta utilización de los indicadores bajo estudio, esto es el resultado de los valores obtenidos para cada uno de sus indicadores, los cuales mostraron alta utilización, a través de la aplicación de técnicas para modelar, gestionar y optimizar los procesos de negocio de la cadena de suministro en las empresas petroleras de fluidos de perforación de la Costa Oriental del Lago en Venezuela.

Tabla 9. Proceso de negocios

\begin{tabular}{|c|c|c|}
\hline Indicadores & $\overline{\boldsymbol{X}}$ & Categoría \\
\hline Administración de las relaciones con el cliente & 4,00 & Alta utilización. \\
\hline Administración del servicio al cliente & 4,03 & Alta utilización \\
\hline Gestión de la demanda & 3,79 & Alta utilización \\
\hline Cumplimiento de los pedidos & 3,80 & Alta utilización \\
\hline Gestión del flujo de fabricación & 4,20 & Alta utilización \\
\hline Desarrollo y comercialización del producto & 3,85 & Alta utilización \\
\hline Devoluciones & 4,10 & Alta utilización \\
\hline Total & 3,97 & Alta utilización \\
\hline
\end{tabular}

Los resultados para la dimensión de alta utilización, alcanza a validar lo expuesto por la Council Logistic Management (2007), donde se expresa que el éxito de la cadena de suministro requiere cambiar las actividades funcionales por actividades integrales relacionadas con los procesos determinantes de la cadena. Aunado a ello, Markovic y Pereira (2007), expresan que el proceso de negocio en la cadena de suministro, es la forma natural de organización, que trata de conseguir que con el trabajo mancomunado y funcional se capturen los requerimientos del negocio para obtener un mejor entendimiento, esto facilita la comunicación así como identificar las mejoras en los procesos.

De manera similar se consigue congruencia con lo expuesto en la investigación, al considerar que la cadena de suministro y los procesos de negocio, enmarcan componentes que deben recibir atención, abarcando una amplia gama desde estratégico hasta operacional, flujo físico hasta flujo de información, estructuras tangibles hasta las estructuras organizacionales y culturales, aspectos que deben ser considerados al ubicarse la dimensión en alta utilización y no en muy alta utilización. 


\section{CONCLUSIONES}

$\mathrm{E}$ n cuanto al proceso de negocios de la cadena de suministro utilizado en las empresas petroleras de fluidos de perforación de la Costa Oriental del Lago, es concluyente que los manejos de acciones en pro de las relaciones y el servicio con el cliente se realizan sin profundizar del todo en las características del segmento, comportamiento de consumo entre otros, sin embargo, las empresas bajo estudio suelen tomar en cuenta las exigencias del consumidor para las mejoras de su servicio.

Para la gestión de la demanda el cumplimiento de esta se observó una brecha entre lo que demanda el mercado y lo que se produce, hecho que a pesar de la posibilidad de las empresas de cumplir cabalmente al mercado no se realiza al máximo de lo esperado (muy alta utilización), es por ello que el cumplimiento de los pedidos se realiza en función de lo que se esté produciendo y le permite mantener un equilibrio entre oferta y demanda.

Por otra parte, se determinó un proceso común en la gestión de los flujos de fabricación que va desde producir, almacenar y entregar los productos terminados al sistema de distribución de acuerdo con las previsiones históricas, realizando para ello inversiones en creación de nuevos procesos de perforación. Finalmente se evidencio en la gestión de devoluciones un proceso en cual toma papel fundamental la empresa, en el que la mayoría de los casos sume las pérdidas por devoluciones.

\section{REFERENCIAS}

Alet, J. (2007). Marketing Directo e Interactivo. ESIC Editorial. Primera edición. Madrid

Castellanos, A. (2015). Logística comercial internacional. Segunda edición. Editor Universidad del Norte. Barranquilla. Colombia

Chase, R., Aquilano, N. y Jacobs F. (2005). Administración de la Producción y Operación para una ventaja Competitiva. Décima Edición. Editorial McGraw Hill. México D.F, México

Council of Logistics Management (2007). Annual Conference, Oak Brook, IL: Council of Logistics Management. Disponible en: http://cscmp.org/

Cox, R. (2012). Servicio al cliente en la cadena de abastecimiento: una oportunidad de negocios. Disponible

en: http://www.revistalogistec.com/index.php/visionempresarial/385 analisis/1137-servicio-al-clienteen-la-cadena-de-abastecimiento-una

Coyle, J.; Langley, J.; Novak, R., y Gibson, B. (2013). Administración de la cadena de suministro. Una
Perspectiva Logística. Novena edición. Cengage Learning Editores, S.A. de C.V. España

Greenberg, P. (2008). Gestión de relaciones con los clientes. Primera edición. McGraw-Hill. Interamericana de España. Madrid

Heizer, J. y Render, B. (2004). Principios de Administración de Operaciones. Quinta edición. Prentice Hall Hispanoamericana. Madrid

Kotler, P. y Armstrong, G. (2012). Fundamentos de marketing. Octava edición. Pearson Education. México

Lambert, D. (2008). Supply Chain Management: Processes, Partnerships, Performance, 4th Edition, Sarasota

Markovic, I. y Pereira, A. (2007). Towards a formal framework for reuse in business process modelling, Actas de 5th International Conference on Business Process Management, 484-495, Brisbane, Australia. Disponible: http://www.scielo.cl/scielo.php?script=sci_nlinks\&r 
ef $=2780610 \&$ pid $=S 0718$ -

$0764200900020000500019 \& \operatorname{lng}=\mathrm{es}$

Miranda, J. (2008). Gestión de Proyectos. Identificación, formulación, evaluación. Quinta edición. MM Editores, Bogotá

Stanton, W.; Etzel, M., y Walker, B. (2004). Fundamentos de mercadotecnia. Décima tercera edición. Editorial McGraw-Hill. México
Vollmann, T.; Berry, W.; Clay Whybark, D. y Jacobs, R. (2005). Planeación y control de la producción: administración de la cadena de suministros. Quinta edición. McGraw-Hill. México 